\title{
RESEARCH HIGHLIGHT Chemical choreography of germinal center B-cell migration
}

\author{
Vu N. Ngo ${ }^{1}$ and Markus Müschen ${ }^{1}$ \\ Cell Research (2019) 29:514-515; https://doi.org/10.1038/s41422-019-0170-7
}

\begin{abstract}
Germinal center (GC) B cells are confined at the center of the B cell follicles by a mechanism that is only partially understood. Using biochemical fractionation and mass spectrometry, Lu and colleagues now identify a novel cue for GC B-cell positioning and define the gradient formation mechanism for this new ligand.
\end{abstract}

During affinity maturation in germinal centers (GCs), B cells and follicular helper $\mathrm{T}$ (Tfh) cells cluster around antigen-loaded follicular dendritic cells (FDCs) in the center of the B cell follicle ${ }^{1,2}$. The positioning of these cells is essential for affinity maturation and requires the sphingosine-1-phosphate receptor S1PR2, which inhibits cell migration upon binding to the cognate ligand S1P ${ }^{3}$. S1P forms a gradient with the lowest concentration at the center of the $B$ cell follicle and the highest concentration in blood and lymph $^{3}$. High expression of S1PR2 on GC B cells and Tfh cells is thought to prevent these cells from migrating to S1P-high areas ${ }^{3-5}$. This mechanism, however, remains incompletely understood, as GC B cells in S1pr2-deficient mice can still be found in the follicular center. This observation subsequently led to the discovery of a second migration inhibitory receptor $\mathrm{P} 2 \mathrm{RY} 8^{5,6}$; however, the ligand of this receptor was not known. In a remarkable tour de force to identify the elusive second ligand, Lu and colleagues ${ }^{7}$ have now identified the cue for $\mathrm{P} 2 \mathrm{RY} 8$, a previously unrecognized molecule composed of glutathione conjugated to a lipophilic isoprenoid geranylgeranyl unit-an intermediary generated in the mevalonate pathway. Lu et al. also defined the mechanism of gradient formation for the new ligand. Their findings highlight an ingenious search for an important intercellular signaling molecule in the milieu of the body interstitial fluids and illuminate a potential link between lipid metabolism and adaptive immune responses.

Similar to S1PR2, the orphan G protein-coupled receptor P2RY8 is highly expressed in GC B cells and signals through the $\mathrm{G}$ protein Ga13. The obscure function of this pathway became recently the focus of mechanistic studies when frequent deleterious mutations of P2RY8, S1PR2 and GNA13 (the gene encoding Ga13) were identified in GC-derived B cell lymphomas, including GCB-DLBCL and Burkitt's lymphoma ${ }^{5}$. While point mutations in $\mathrm{P} 2 \mathrm{RY} 8$ are common in GC-derived $B$ cell lymphomas, the coding exons of P2RY8 are frequently deleted by $P 2 R Y 8-C R L F 2$ gene rearrangements in B-lineage acute lymphoblastic leukemia. P2RY8-CRLF2 rearrangement results from an interstitial microdeletion involving the short arm of both sex chromosomes $(\operatorname{del}(X)(p 22 p 22)$ or $\operatorname{del}(Y)(p 11 p 11)$. This deletion results in loss of P2RY8 function and overexpression of the CRLF2 cytokine receptor chain by placement of the CRLF2 coding sequence under transcriptional control of regulatory elements of $\mathrm{P} 2 \mathrm{RY} 8^{8}$. Deletions and amplifications of $\mathrm{P} 2 \mathrm{RY} 8$ are frequent in solid tumors as well, however, the functional significance of these lesions is not clear.
Consistent with a function of $\mathrm{P} 2 \mathrm{RY} 8$ as an additional migrationinhibiting receptor, like S1PR2, the authors observed that the loss of GC B-cell confinement only occurred in Ga13-, but not S1pr2-, deficient mice ${ }^{5}$. Genetic evidence for a role of P2RY8 in the regulation of GC B-cell positioning could be derived from a knockout mouse model, however, while P2RY8 is conserved among vertebrates, mice are lacking a P2RY8 ortholog. In gain-offunction studies, Muppidi et al. overexpressed P2RY8 to test its influence on GC B-cell localization ${ }^{5}$. Strikingly, like S1PR2, overexpression of P2RY8 suppressed GC B-cell growth and migration in mouse lymph nodes and Peyer's patches ${ }^{5}$. Furthermore, P2RY8expressing B cells are localized within the follicular center while cells carrying empty vector controls are found throughout the follicles ${ }^{5}$. These remarkable findings indicate a dual role for the human P2RY8 in regulating the growth and positioning of GC B cells. To complete the regulatory circuit of GC B-cell localization, Lu et al. $^{7}$. in the present study set out to search for the elusive P2RY8 ligand. The authors designed an elegant bioassay based on the concept that engagement of P2RY8 by its cognate ligand would suppress migration towards chemoattractant cues (e.g., CXCL12 and CXCL13). Among extracts from various mouse tissues, the highest bioactivity was found in the bile and appeared to be an amphiphilic molecule. Further clues were derived from the finding that statins, but not other lipid biosynthesis inhibitors, suppressed the bioactivity, which could be rescued by products of the mevalonate pathway such as mevalonate and geranylgeranyl pyrophosphate. Importantly, mass spectrometry signals dropped out from the statin-treated samples, which were included to specifically screen for products from the isoprenoid biosynthesis pathway. Using tandem mass spectrometry, a candidate compound was identified and confirmed as a conjugate between geranylgeranyl ion and glutathione (GGG). The chemical composition of the new ligand was not previously described, however, glutathione-conjugated lipids such as leukotriene $\mathrm{C}_{4}\left(\mathrm{LTC}_{4}\right)$ are potent metabolites that bind their cognate $G$ protein-coupled receptors and play important roles in inflammation and cell migration ${ }^{9}$. Importantly, like S1PR2, which represses AKT activation, GGG also inhibits AKT phosphorylation in P2RY8-transduced B-cell lines while it fails to antagonize chemokine-induced AKT activation in P2RY8-deficient cells. These results and other convincing evidence support the conclusion that the new ligand GGG acts through P2RY8 to inhibit chemokine-induced migration and AKT activity (Fig. 1).

Previous work from Jason Cyster's laboratory demonstrated that S1PR2 prevents GC B cells from migrating towards increasing S1P concentrations in the outer follicle ${ }^{3,5}$. In analogy to S1PR2, the authors studied how GGG gradients in lymphoid tissues regulate P2RY8 function and its ability to suppress migration. These experiments revealed that the glutathione conjugate plays a central

${ }^{1}$ Department of Systems Biology and City of Hope Comprehensive Cancer Center, Monrovia, CA 91016, USA

Correspondence: Markus Müschen (mmuschen@coh.org)

Published online: 25 April 2019 


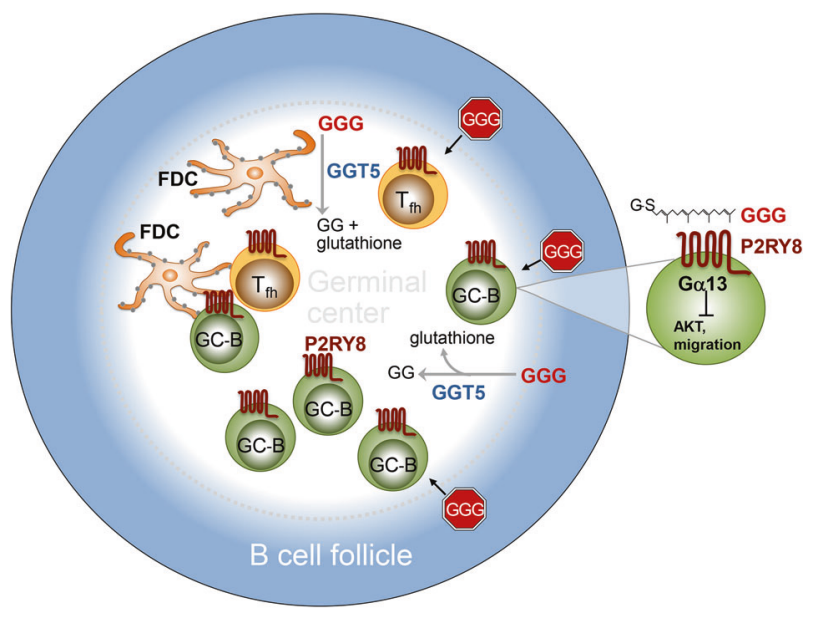

Fig. 1 The novel ligand geranylgeranyl glutathione (GGG) orchestrates GC B-cell positioning. GGG mediates the confinement of GC B and Tfh cells in the follicular center through binding to the Go13dependent receptor P2RY8, resulting in AKT inactivation and inhibition of cell migration towards GGGhigh regions. The enzyme GGT5, produced by GC FDCs, metabolizes GGG into an inactive form, thus reducing GGG concentrations in the center of the B-cell follicles. GC germinal center, Tfh follicular helper T cells, FDC follicular dendritic cells, pAKT phosphorylated AKT

role in GGG metabolism and, possibly, its gradient formation. Indeed, the authors identified the $\gamma$-glutamyltransferase GGT5 as a negative regulator of GGG bioactivity by cleaving off its $\gamma$-glutamyl group. Using in situ hybridization and single-cell RNA-seq, the authors convincingly demonstrated that the tissue distribution of GGT5 provides a network of cell types that regulate the GGG-P2RY8 pathway by forming a follicle-specific GGG gradient. GGT5 is highly expressed in a pattern coinciding with $\mathrm{CR}^{+} \mathrm{GC}$ FDCs, but not in GC $B$ cells, and minimally expressed in the outer areas of the $B$ cell follicles. In this way, the GC FDCs participate as a "sink" to reduce GGG concentrations in the follicular center, hence generating a lowto-high gradient towards the outer regions of the follicles. The new findings support a model in which GGG acts as a signaling cue to inhibit P2RY8-expressing GC B cells from expansion beyond the GC boundary. With the remarkable discovery of GGG as the new ligand for P2RY8, Lu and colleagues introduce a new class of polar lipids that play an important role in the T-dependent antibody responses.

\section{ADDITIONAL INFORMATION}

Competing interests: The authors declare that they have no conflict of interest.

\section{REFERENCES}

1. Liu, Y. J., Zhang, J., Lane, P. J., Chan, E. Y. \& MacLennan, I. C. Eur. J. Immunol. 21 2951-2962 (1991).

2. Cyster, J. G. et al. Immunol. Rev. 176, 181-193 (2000).

3. Green, J. A. et al. Nat. Immunol. 12, 672-680 (2011).

4. Moriyama, S. et al. J. Exp. Med. 211, 1297-1305 (2014).

5. Muppidi, J. R. et al. Nature 516, 254-258 (2014).

6. Muppidi, J. R., Lu, E. \& Cyster, J. G. J. Exp. Med. 212, 2213-2222 (2015).

7. Lu, E., Wolfreys, F. D., Muppidi, J. R., Xu, Y. \& Cyster, J. G. Nature 567, 244-248 (2019).

8. Yoda, A. et al. Proc. Natl Acad. Sci. USA 107, 252-257 (2010).

9. Peters-Golden, M., Gleason, M. M. \& Togias, A. Clin. Exp. Allergy 36, 689-703 (2006). 